\title{
Die Rückeroberung der Vergangenheit: Istanbuls visuelle Kommunikation zum Europäischen Kulturhauptstadtjahr 2010
}

\author{
Johannes Marent
}

(C) The Author(s) 2016. This article is available at SpringerLink with Open Access

Zusammenfassung Im Rahmen des global geführten Wettbewerbs der Städte um Wissensarbeiterinnen, Touristen und Investoren intensiviert sich das Interesse am „Bild“ der Stadt. Stararchitektinnen und Marketingexperten sollen die Besonderheiten stilisieren und die Orte visuell unterscheidbar machen. Mit Bezug auf Anselm Strauss wird auch aus soziologischer Sicht auf die Bedeutung medialer Stadtbilder hingewiesen. Sie erzeugen Vorstellungen vom „Ganzen der Stadt“, schaffen Orientierung im Alltag und strukturieren Diskurse (Strauss 1961). Gegenstand der Analyse ist die Werbekampagne „Istanbul 2010. Europäische Kulturhauptstadt“. Das Werbesujet wird dabei als „städtisches Selbstbild“ verstanden, als eine Kommunikationsofferte, wie die Stadt gesehen werden möchte. Die vom Stadtmarketing kreierten Images, so die These, schließen zum einen an innerstädtische Diskurse an und nehmen zum anderen auch Bedeutungsverschiebungen vor. Für Stadtbilder gilt, dass aus einem breiten Pool an möglichen Narrativen und Symbolen (Heterogenität) jeweils bestimmte zu einem mehr oder weniger kohärenten „Bild der Stadt“ verknüpft werden (Verdichtung). Insofern werden Fragen danach relevant, was uns Werbebilder zu Istanbul als Kulturhauptstadt über diese Stadt erzählen und worüber sie schweigen. Auf theoretischer Ebene wird der stadtsoziologische Diskurs „Eigenlogik der Städte“ (Berking et al. 2008; Löw 2008) aufgegriffen. Dieser ermöglicht es, die Stadt als Forschungsobjekt und nicht lediglich als Kontext der Untersuchung zu konzeptualisieren. Die Auswertung eines exemplarischen Werbebildes erfolgte mittels der Segmentanalyse nach Breckner (2010).

Schlüsselwörter Istanbul · Visuelle Soziologie · Stadtsoziologie · Stadtbild · Stadtmarketing

J. Marent $(\square)$

Brandgasse 9/2, 6900 Bregenz, Österreich

E-Mail: johannes.marent@univie.ac.at 


\title{
The recapture of the past: Istanbul's visual communication during the European Capital of Culture event 2010
}

\begin{abstract}
In the context of the global competition to attract knowledge-workers, tourists, and investors, cities work carefully on their iconic representation. Star architects and marketing experts try to strengthen the peculiarities of cities and stage their distinctiveness. Following Anselm Strauss, city images play a significant role in structuring the imagination of the city "as a whole". They help to organize everyday life in urban settings and structure local discourses (Strauss 1961). The object of analysis is the image campaign "Istanbul 2010: European Capital of Culture". The advertising motif is considered as a "self-portrait" of the city, a communicative strategy how the city wants to be seen and understood. The argument is, that the city images select from a great pool of possible narratives and symbols (heterogeneity) certain aspects and construct a coherent and particular "image of the city" (densification). In this respect the question has to be raised what the image depicts and what it conceals. The article refers to the current debate within the field of urban sociology about the "intrinsic logic of cities" (Berking et al. 2008; Löw 2008). Thereby the "city" is conceptualized as the object of investigation rather than merely serving as the analytic context. To evaluate the advertising image a segmentanalytical approach is employed (Breckner 2010).
\end{abstract}

Keywords Istanbul · Visual sociology · Urban sociology · City image · City marketing

\section{Einleitung}

Als Reaktion auf den post-industriellen Strukturwandel differenzierte sich Anfang der 1980er-Jahre das Stadtmarketing als Subsystem der Stadtökonomie heraus. Die urbanen Zentren mussten sich auf neue wirtschaftliche Standbeine stellen und sehen sich seither in einem weltweiten Konkurrenzkampf um Wissensarbeiterinnen ${ }^{1}$, Investoren und Touristinnen. Dieser Wettbewerb wird visuell geführt (Löw 2008), es gilt die Unverwechselbarkeit der Orte zu sichern und gleichzeitig Wiedererkennbarkeit zu schaffen. Das Stadtmarketing verknüpft dabei architektonische Formen und lokale Narrative zu einem kommunizierbaren Image der Stadt.

Der folgende Beitrag greift eine solche Imagekommunikation auf. Anhand eines konkreten Beispiels - Istanbuls Werbekampagne zum Kulturhauptstadtjahr 2010 wird gezeigt, dass derlei visuelle Imageprodukte eine zwar populäre, aber doch hoch verdichtete Bildsprache entwickeln. In dem hier zur Analyse herangezogenen Werbebild (Abb. 1) werden drei differente Bedeutungsstrukturen zu einem kongruenten Image verknüpft: (1) Die Kampagne richtet sich an internationale Touristen und versucht deren Vorstellungen über die Stadt zu bestätigen. (2) Gleichzeitig ist sie das Produkt einer lokalen und nationalen Deutungselite. Die Präsentation Istanbuls

\footnotetext{
1 Zum Zwecke einer gendersensiblen Sprache wird im Folgenden alternierend zwischen der männlichen und weiblichen Form gewechselt. Es sollten sich in jedem Fall alle angesprochen fühlen.
} 
als Kulturhauptstadt Europas wurde von der Politik vereinnahmt und zur Machtdemonstration benutzt. (3) Abgesehen davon greift die Werbekampagne innerstädtische Diskurse und den städtischen Symbolkosmos auf und kann in diesem Sinne als städtisches Selbstbild verstanden werden.

Theoretisch wird an den stadtsoziologischen Diskurs „Eigenlogik der Städte“ (Berking et al. 2008; Löw 2008) angeschlossen. Städte werden dabei als „Sinneinheiten" verstanden, die jeweils ganz spezifische Symbolsysteme ausbilden und in Bildern, Erzählungen und Lebensweisen Ausdruck finden und verhandelt werden. In diesem Sinne dient Istanbul der folgenden Analyse nicht nur als Kontext, in dem die visuelle Kommunikation eines Events untersucht wird. Vielmehr ist die Stadt und die mit ihr verknüpften Vorstellungswelten Gegenstand der Untersuchung. Des Weiteren wird auf die kultursoziologische Bedeutung der Analyse von Werbeformaten und insbesondere Stadtmarketingimages eingegangen. Es wird argumentiert, dass ein Verständnis, das Stadtmarketingimages lediglich als banale und problemlos dechiffrierbare Botschaften betrachtet, zu kurz greift. Ebenso einseitig ist die Annahme, dass derlei Images gänzlich von der lebensweltlichen Wirklichkeit abgekoppelt sind und die Deutungseliten uneingeschränkte Freiheiten bei ihrer Gestaltung haben.

Empirisch wird das bildanalytische Verfahren der Segmentanalyse (Breckner 2010) herangezogen. Anhand einer materialen Analyse wird gezeigt, wie Sichtbarkeit, d.h. die Präsenz des Bildes, in einem konkreten sozialen Zusammenhang Wirkung entfaltet. Das Bedeutungspotenzial des hier analysierten Bildes wird dementsprechend weder auf das mediale Genre „Stadtmarketing“ eingeschränkt noch auf die Entstehungs- und Produktionsbedingungen reduziert. Bilder, so die hier vertretene Position, sind weder lediglich Abbilder der Wirklichkeit noch Simulacren, die gänzlich gesteuert und kontrolliert werden können. Bilder entfalten ihr Bedeutungspotenzial in der kommunikativen Verwendung.

\section{Urbane Vorstellungswelten}

Als eine der ersten sozialwissenschaftlichen Studien, die visuelle Stadtbilder und die ihnen zugrundeliegenden Vorstellungswelten untersucht, kann das Werk ,Images of the American City“ von Anselm Strauss (1961) angeführt werden. Für Strauss war die Stadt nicht nur eine physische Gestalt, sondern vor allem eine symbolisch aufgeladene Form, eine Sinneinheit, die in bildlichen Stadtdarstellungen in verdichteter Prägung zu analysieren ist. Ihn interessierten die Unterschiede zwischen den Darstellungen verschiedener Städte und wie sich der jeweilige Symbolkosmos etablierte. Folgt man Strauss, so nehmen Stadtbilder eine Verdichtungsleistung vor, durch die die Stadt als Ganzes - ,the city as a whole“ - perzipiert werden kann. Die Stadt tritt damit als distinktive Sinneinheit, als ein vorstellbares Ganzes, das mit spezifischen Qualitäten und Attributen ausgestattet ist, in den Fokus der soziologischen Analyse. Die Wirkmächtigkeit urbaner Vorstellungswelten wird vom stadtsoziologischen Diskurs „Eigenlogik der Städte“ (Berking et al. 2008; Löw 2008) erneut hervorgehoben. Der Eigenlogikansatz konzipiert die Stadt als raumstrukturelle Form, die vor allem durch Dichte und Heterogenität gekennzeichnet ist. Für Stadtbilder gilt, dass aus einem breiten Pool an möglichen Narrativen und Symbolen (Heterogeni- 
tät) jeweils bestimmte zu einem mehr oder weniger kohärenten „Bild der Stadt“ verknüpft werden (Verdichtung). Damit tritt die Stadt als Sinneinheit hervor. Derlei Vorstellungswelten sind nicht bloß „fiktiv“, sondern beeinflussen lokale Praktiken und Diskurse. Denn, ,[a]ls von Geschichte und Geschichten durchtränkter, kulturell kodierter Raum bildet die Stadt einen Vorstellungsraum, der den physikalischen insofern überlagert, als er der durch die begleitenden Bilder und Texte hindurch erlebte und erfahrene Raum ist" (Lindner 2008, S. 86; Hvh. i. O.). Hier wird auf das Interferenzverhältnis verwiesen, das der Erforschung bildlich vermittelter Vorstellungswelten soziologische Relevanz verleiht. Die Sinneinheit Stadt überlagert ihre Materialität und schreibt sich auch in die Praktiken ein. „Texte und Bilder gehen in Praktiken der Architektur, Raumplanung und Raumnutzung ein, diese wiederum wirken zurück auf die Bilder und Texte, die über eine Stadt kursieren“ (Wietschorke 2013, S. 205). Die Aufgabe der soziologischen Analyse besteht darin, dieses Wechselverhältnis zu beschreiben. Einerseits gilt es, die Vorstellungswelten zu erschließen, die den städtischen Repräsentationen zugrunde liegen, andererseits, die Herstellung eben dieser zu beleuchten und sie in ihrem Konstruktionscharakter zu erfassen. In diesem Sinne gilt es folgende Fragen zu beantworten: Inwiefern schließt das hier untersuchte Werbebild an städtische Narrative und Symboliken an? Welche Umschreibungen werden vorgenommen? Welche neuen Vorstellungen entstehen durch diesen „Interpretationsprozess“? Und: welche Aushandlungsprozesse stehen hinter dieser Deutung der Stadt?

\section{Inszenierte Wirklichkeiten}

Bevor der konkrete Analysegegenstand vorgestellt wird, soll das mediale Genre „Werbung“ hinsichtlich seiner gesellschaftstheoretischen Relevanz reflektiert werden. Werbung, so die verbreitete Annahme, würde die Wirklichkeit eher verschleiern als zum Ausdruck bringen (Zurstiege 2007, S. 27). Hier wird eine konträre kultursoziologische - Perspektive eingenommen. Diese geht erstens davon aus, dass Werbung sich des Symbolhaushalts der Kultur bedient (Müller-Doohm 1997, S. 89). D. h., sie greift gesellschaftliche Normen, Stereotypen und bestimmte Stilisierungen auf. Zweitens stellt sie diese nicht einfach nur dar, sondern nimmt Umdeutungen vor, die wiederum vom gesellschaftlichen Diskurs aufgegriffen werden. Die Werbung liefert mit Bildern von der Welt gleichzeitig ,Weltbilder“ (Berger 1974; Willems und Kautt 2003, S. 19). Vor allem wegen des hohen Verbreitungsgrades und der wirklichkeitskonstituierenden Wirkung sind Werbeformate für die kultursoziologische Analyse von Bedeutung.

Um „Inszenierung“ und gesellschaftliche „Wirklichkeit“ nicht als widersprüchliche Kategorien zu verstehen, wird auf Überlegungen von Erving Goffman zurückgegriffen. Für Goffman, der für seine „Bühnenmetapher“ bekannt ist, ist jede Alltagssituation von einer (Selbst-)Inszenierung geprägt. Die „Inszenierung“ gehört zur „Natur“ der Gesellschaft. Es ist nicht der Gegensatz von Wirklichkeit und Inszenierung, der die Werbung vom Alltagshandeln unterscheidet. Vielmehr erkannte Goffman in Werbebildern eine Ritualisierung von sozialen Idealen, die er wegen der genretypischen Standardisierung, Übertreibung, Vereinfachung und Ausklam- 
merung als „Hyper-Ritualisierung“ beschreibt (Goffman 1981). Werbung legt nicht einen „Schleier“ über die Wirklichkeit, vielmehr bringt sie gesellschaftliche Werte kondensiert zum Ausdruck. Goffmans Prämissen sollen hier für die Analyse von Stadtmarketingimages übernommen werden. Dabei wird insbesondere auf seinen interaktionstheoretisch gefassten Imagebegriff zurückgegriffen. Goffman beschreibt Image als ,ein in Termini sozial anerkannter Eigenschaften umschriebenes Selbstbild, - ein Bild, das die anderen übernehmen können“ (Goffman 1986, S. 10). Diese Images sind variabel. Je nach sozialem Kontakt wird eine bestimmte Strategie gewählt. Man gibt sich etwa vor seinem Arbeitgeber etwas seriöser als vor seinen Freunden abends in der Kneipe. Auch das Stadtmarketing segmentiert zunächst die Zielgruppen, um dann zu entscheiden, ob Investoren, Konferenzreisende, qualifiziertere Arbeitskräfte, Touristen usw. mit demselben Image beworben werden sollen. Dennoch gilt für die Kommunikation des Stadtmarketings wie für die Faceto-face-Interaktion: Man muss bis zu einem gewissen Grad ,,verkörpern“, was man vorgibt zu sein. Goffman spricht etwa von einem „falschen Image“, wenn Informationen über eine Person, ,ans Licht gebracht werden, die selbst mit größter Mühe nicht in die von ihm verfolgte Strategie integriert werden können“ (ebd., S. 13). Für die Images des Stadtmarketing gilt ähnliches: „Keiner Imageproduktion ist es gegeben, das Bild einer Stadt jenseits und unabhängig der spezifischen kumulativen Textur dieser Stadt erfolgreich zu stabilisieren“ (Berking und Schwenk 2011, S. 23).

Ein vom Marketing verbreitetes Stadtimage ist damit eine komplexitätsreduzierende Kommunikationsofferte, die die Vorstellung über die Stadt beeinflusst. Sie greift bestimmte, bereits fluktuierende Vorstellungen über die Stadt auf und nimmt Neuinterpretationen vor. Dabei kommt es zu Bedeutungsverschiebungen und zur Steigerung der Diversität durch ,neue“ Bilder. Das Stadtmarketing ist eine Schnittstelle, an der unterschiedliche Sichtweisen ausgehandelt werden. Das nach außen kommunizierte Image braucht die innerstädtische Identifikation. Die Konsensfindung darüber, wie die Stadt dargestellt werden soll, ist oft ein langwieriger Prozess. Häufig ist eine ,corporate identity“ nicht möglich bzw. auch nicht erwünscht, weshalb in manchen Städten verschiedene Akteure im Feld des Stadtmarketings unterschiedliche Images verbreiten (Richter 2014). Dass das Stadtmarketing nicht unabhängig vom innerstädtischen Diskurs agiert, wird daran deutlich, dass Imagekampagnen vielfach und eingehend in den lokalen Medien diskutiert und kritisiert werden. ${ }^{2}$ Anders als bei der Produktwerbung kann sich die Stadt - d. h. die Bevölkerung auch gegen das ihr diktierte Image zur Wehr setzen. Das Stadtmarketing kann als zweifacher Aushandlungsprozess beschrieben werden: Global muss sich das Image der Stadt gegenüber anderen Städten durchsetzen und Differenz behaupten. Lokal gilt es, den weitest möglichen Konsens zu finden und Identifikation zu stiften.

\footnotetext{
2 Zu diesem Befund kam das DFG-Projekt „Die Inszenierung des Ganzen: Stadtmarketing und die Eigenlogik der Städte“ (2011-2014); siehe (Frank et al. 2014).
} 


\section{Die Inszenierung Istanbuls als Kulturhauptstadt Europas}

Die nachstehende Rekonstruktion eines Werbebildes basiert auf der soziologischen Segmentanalyse nach Roswitha Breckner (2010), die an ikonisch-phänomenologische Bildkonzepte anknüpft. Dabei gilt es, der bildlichen Präsenz Rechnung zu tragen und das Bild nicht im Vorfeld auf bestimmte Bedeutungsinhalte zu reduzieren, die etwa aus dem Wissen über Produktionsbedingungen, den Kontexten der Verwendung, die Stilgeschichte, die Rezeption usw. hervorgehen. Dadurch soll die affektive Wirkung des Bildes rekonstruiert und der unmittelbar wirkenden Suggestivität Aufmerksamkeit gezollt werden. Die Analyse begann mit der Aufzeichnung des Wahrnehmungsprozesses, den das Bild bei der Erstbetrachtung auslöste. Es wurde festgehalten, welche Bildelemente (Segmente) der Blick in welcher Abfolge wahrnahm und welche Assoziationen und Eindrücke das Bild auslöste. Eine erste Erfassung des formalen Bildaufbaus diente, zusammen mit dem Wahrnehmungsprozess und den Ersteindrücken, der Identifikation von relevanten Bildsegmenten. Die Segmente wurden anschließend sowohl einzeln als auch in ihren Zusammenhängen interpretiert. Durch das Verfahren wurden möglichst viele Bedeutungen zugelassen und Lesarten gebildet, die in einer schrittweise vollzogenen Analyse zu Hypothesen bezüglich der latenten wie manifesten Sinnstruktur des Bildes verdichtet wurden. In einem weiteren Analyseschritt standen perspektivische Eigenheiten, szenische Arrangements und die planimetrische Komposition des Bildganzen im Fokus. Dabei wurde darauf geachtet, wie sich die einzelnen Segmente ins Sinngewebe des Gesamtbildes einschreiben. Erst nach eingehender Analyse der visuellen Präsenz flossen Hintergrund- und Kontextinformationen in die Analyse ein. Das Bild wurde re-kontextualisiert. Es galt, das Wechselspiel zwischen Bildimmanenz und gesellschaftlichem „Rahmen“ (Goffman 1980; Raab 2012), in den das Bild eingebettet ist, zu beschreiben. Die folgende Darstellung erfolgt ergebnisorientiert. Das heißt,

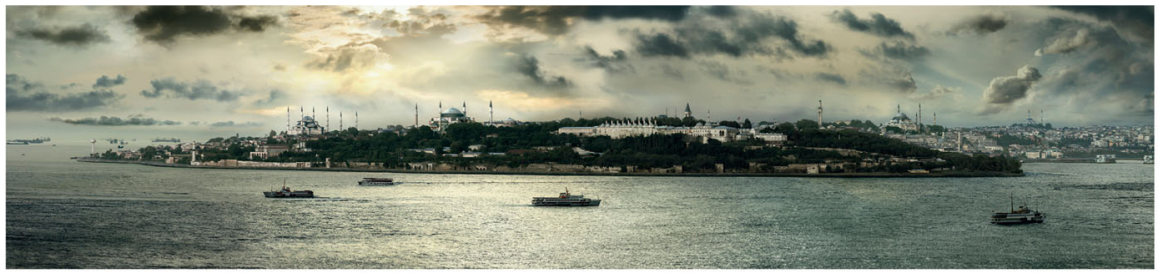

Istanbul... the most inspiring city in the world.

Itrathbul 2010 European Capital of Culture will further inspire you
swath

Bea part of this unique experience.
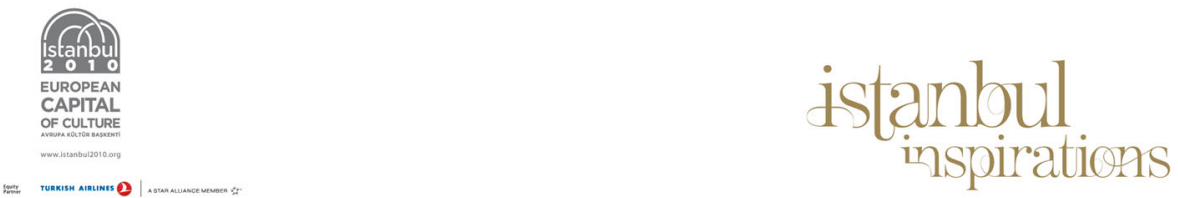

Abb. 1 Motiv Internationale Werbekampagne, European Capital of Culture Agency 


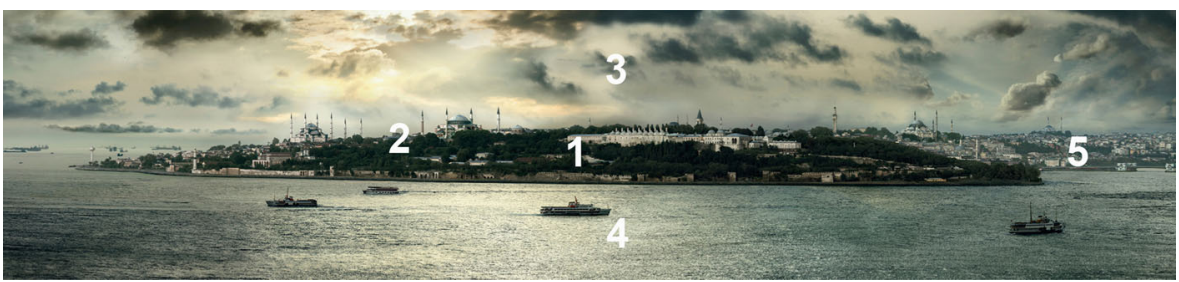

Abb. 2 Wahrnehmungsprozess Bild ohne Bildtext

die Fülle an Differenzierungen und Lesarten wird nicht aufgespannt, sondern gezielt auf die Hauptlinien der Interpretation zugeschnitten. ${ }^{3}$

Das Bild steht am Anfang der nachfolgenden Analyse. Der Leserin wird damit Zeit gegeben, um unbeeinflusst erste subjektive Eindrücke zu registrieren.

\subsection{Wahrnehmungsprozess und Segmentbildung}

Der Wahrnehmungsprozess des Bildes ohne den Bildtext erschloss sich wie folgt: Als erstes erfasste mein Blick die Insel als Ganzes, wobei er sogleich auf die zwei Moscheen fokussierte und kurz auf diesen verweilte, um von dort die einzelnen Gebäude der Insel zu untersuchen. Dies geschah in folgender Reihenfolge: Zunächst erkannte ich das Gebäude etwas rechts von der Mitte der Landmasse, welches ich sofort als Palast kategorisierte. Dann fiel mein Blick auf die weiteren Turmspitzen hinter dem Palast. Von dort glitt er hinab zur Stadtmauer und wanderte diese entlang bis er die verdichtete Siedlung an der linken Spitze der Insel erfasste und schließlich am Leuchtturm Halt machte. Anschließend wanderte mein Blick den Himmel entlang, den ich als dramatisch und außergewöhnlich empfand. Dann erfasste ich das Wasser mit den Schiffen. Zuletzt erkannte ich das stärker verdichtete Stadtgebiet in der rechten Bildhälfte, das perspektivisch hinter der Insel liegt. Die Landmasse in der Bildmitte wurde von mir zunächst als Insel identifiziert. Etwas später realisierte ich, dass es sich sehr wahrscheinlich um eine Landzunge bzw. eine Halbinsel handelt. Meine Erstwahrnehmung wurde im Rahmen mehrerer Interpretationsgruppen ${ }^{4}$ diskutiert und das Bild analysiert. Bezüglich des Ersteindruckes fiel auf, dass einige der Teilnehmerinnen wie ich selbst zunächst von der pittoresken Landschaft und den Farben angetan waren, während andere Interpretationsteilnehmer im Bild sofort Machtsymboliken erkannten. Das Bild scheint, ähnlich wie ein Vexierbild, beide Betrachtungsweisen zuzulassen. Auf diesen Aspekt der differenten Erstwahrnehmung wird an späterer Stelle noch ausführlich eingegangen.

\footnotetext{
3 Der gesamte Interpretationsverlauf wird in Marent (2016) vorgestellt.

4 Interpretationsgruppen nutzte ich, um meine eigenen Auslegungen, v. a. den Wahrnehmungsprozess und die Segmentinterpretationen, zu überprüfen und um weitere mögliche Lesarten zu generieren. Anders als ich hatten die Teilnehmerinnen kaum Kontextinformationen, etwa zur Strategie der Kampagne. Die Teilnehmerinnen waren Kolleginnen und Studenten am Institut für Soziologie der Universität Wien sowie Kollegen der DFG-Forschergruppe „Eigenlogik der Städte“, die an der Technischen Universität Darmstadt angesiedelt war; ihnen sei an dieser Stelle herzlich gedankt.
} 


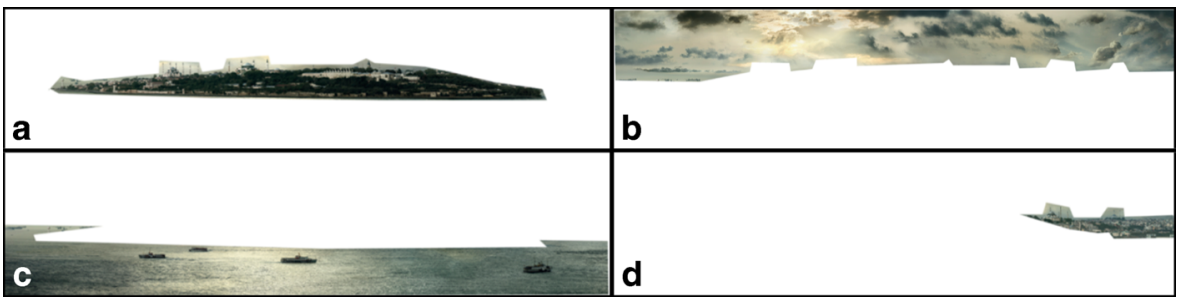

Abb. 3 Segmentbildung. a Segment 1, b Segment 2, c Segment 3, d Segment 4

Aufgrund des zuvor beschriebenen Wahrnehmungsprozesses und einer ersten Beschreibung des Bildaufbaus, der im Wesentlichen durch einen Vorder-, Mittel- und Hintergrund gegliedert ist und eine Zentralperspektive mit Panoramakrümmung suggeriert, wurden die Segmente 1-4 abgebildet (Abb. 3).

\subsection{Analyse der Segmente}

Auf dem ersten Bildsegment sieht man die historische Halbinsel Istanbuls mit dem Topkapi Palast, der Hagia Sophia und der Blauen Moschee. ${ }^{5}$ Betrachtet man die Form dieser Stadtdarstellung, so erkennt man zwei wesentliche Analogien zu den europäischen Profilansichten des 15. Jahrhunderts (De Seta 1999, S. 11; Michalski 1999, S. 46 ff.; Günther 2009, S. 32 ff.): erstens der flächige Aufbau der Stadt mit der hierarchischen Überhöhung der Sakralbauten, gefolgt von dem ,,weltlichen“ Machtzentrum; zweitens die Ummauerung der Stadt, die sie zusammen mit dem stufenweisen Aufbau als Ellipse erscheinen lässt. Die Form der Ellipse scheint als eine Art Ideogramm zu funktionieren, das die Idee der antiken Polis aufnimmt. Die Form visualisiert sozusagen die Stadt als geschlossene Einheit (Michalski 1999, S. 49): Sie zeigt, im Sinne der antiken Polis, eine eigenständige politische - in diesem Fall vor allem eine religiöse - Stadt, die von Gleichheit und Übereinstimmung geprägt ist, wodurch Unterschiede bzw. Heterogenität (politische, religiöse) unter die hegemoniale Macht subsumiert werden.

Der malerische Himmel, der durch Wolken, Licht und Farben Stimmung erzeugt, konstruiert einen Vergangenheitsbezug. Auf den ersten Blick ist schwer zu entscheiden, ob es sich um eine Fotografie oder ein Gemälde handelt. Die Farben und die Dramatik des Himmels erinnern an die Romantik des ausgehenden 18. Jahrhunderts. Zu nennen wäre etwa Caspar David Friedrich mit seinen von metaphysischtranszendenten Stimmungen geprägten Landschaftsmalereien. Die atmosphärischen Naturdarstellungen der Romantik sollten dem Betrachter bewusst machen, dass der Mensch höheren Mächten ausgeliefert ist. Die hier präsentierten dunkeln Wolken verweisen auf Unwetter und Naturgewalten. Das weiße Licht wiederum, das durch sie hindurch dringt und sich über den zwei Moscheen bündelt (siehe Abb. 7), erzeugt eine sakrale Stimmung. Es ist ein stilistischer Verweis auf ,göttliche Macht“, von der diese Stadt umhüllt bzw. geschützt ist.

\footnotetext{
5 Um die Analyse zu straffen, werden einige Hintergrundinformationen bereits preisgegeben.
} 


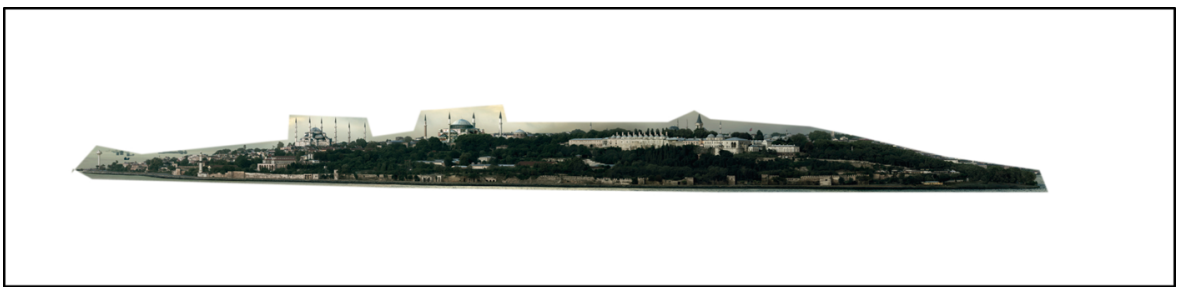

Abb. 4 Segment 1 „Halbinsel“

Über das im Vordergrund auftauchende Wasser und die Schiffe wird die Stadt ins Verhältnis zur Welt gesetzt und als globales Machtzentrum erkennbar. Die topologische Dimension der Lage am Meer relativiert die „Introvertiertheit““, die die Darstellung der Stadt in Form einer Ellipse suggerierte. Die Schiffe im Bildvordergrund verweisen auf Handel und Tourismus. Der Segmentzusammenhang von Stadt auf der Halbinsel und Wasser demonstriert Einheit nach innen und Offenheit nach außen.

Über die szenische Choreographie des Bildes werden drei Machtsymboliken in Beziehung gesetzt: (1) Hegemoniale Macht: die Stadt wird als politische Einheit präsentiert, (2) göttliche Macht: symbolisiert durch die sakrale Stimmung des Himmels und (3) weltliche bzw. wirtschaftliche Macht: symbolisiert durch die Konnektivität des Wassers.

\subsection{Analyse der Textelemente}

Bei der Interpretation der Textsegmente beginne ich mit dem Schriftzug direkt unterhalb des visuellen Segments. Nachdem man das Wort „Istanbul“ ausspricht, bedarf es zunächst einmal einer Pause, visualisiert durch drei Punkte und eine Abstufung. Das Wort „Istanbul“ allein, so kann aufgrund der „Pause“ gefolgert werden, ruft schon Bilder bzw. Assoziationen hervor. Etwa: Bosporus, lange Geschichte, Konstantinopel, osmanisches Reich, Brücke zwischen „Ost“ und „West“, Tradition und Moderne, Bevölkerungswachstum, Wirtschaftsmacht, Inspirationsquelle zahlreicher europäischer Schriftsteller und Reisender, kulturelle Vielfalt, hegemoniale Machtansprüche etc. Anhand des Folgesatzes ist erkennbar, dass es sich um eine „Stadtwerbung“ für Istanbul handelt, welche die Stadt als ,the most inspiring city in the world“" anpreist. Istanbul misst sich damit nicht mit anderen Städten der Türkei und auch nicht ,nur“ mit Städten Europas, sondern mit der ganzen Welt. Das Wort ,,inspiring“ wird mit dem Superlativ versehen. Auffällig am Folgesatz ist, dass nicht die ,eigentlichen“ Inspirationsquellen aufgezählt werden, sondern gleich ,zusätzliche“ (,further“) Quellen der Inspiration - ,contemporary art“ und ,urban culture“ aufgezählt werden. In Kombination mit dem Beginn des ersten Satzes, dem Namen der Stadt gefolgt von einer Pause, lässt sich vermuten, dass es allgemein bekannt sein müsste, weshalb Istanbul ,inspiriert“ und lediglich ,zusätzliche“ bzw. „,neue“ Quellen der Inspiration angegeben werden müssen. Des Weiteren wird die Kontextinformation gegeben, dass Istanbul 2010 Europäische Kulturhauptstadt ist, und die Betrachterin wird aufgefordert, an dieser einzigartigen Erfahrung (,,unique experi- 


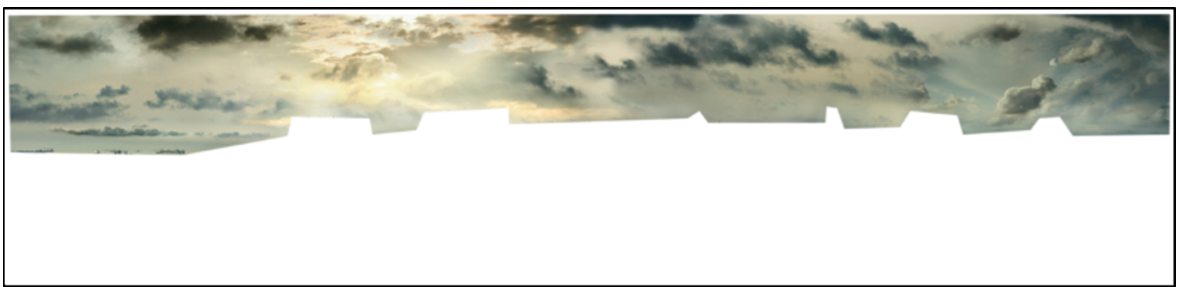

Abb. 5 Segment 2 ,Himmel“

ence") teilzuhaben. Dadurch wird ersichtlich, dass sich die Botschaft „nach außen“ richtet, an internationale Touristen, internationale Künstlerinnen etc. Die Aufforderung adressiert Leute, die nicht in dieser Stadt leben, ansonsten wären sie schon ein Teil dieser Erfahrung. Es wird ein schlichter und moderner Schrifttyp verwendet (ohne Hervorhebung, geradlinig und ohne Schnörkel). Die mattgoldene Farbe des oberen Schrifttyps vermittelt Kostbarkeit.

Die drei Rundbögen über dem Schriftzug „Istanbul 2010“ erinnern an Arkaden bzw. an Kuppeln. Im Falle Istanbuls und vor allem in Kombination mit dem zuvor interpretierten visuellen Segment ist ersichtlich, dass diese Rundbögen die Kuppeln von Moscheen symbolisieren. Die Farbgestaltung sowie die geradlinigen Schriftzüge können als ,gegenwärtig“ bzw. „modern“ bezeichnet werden. Die Farbe ist wenig aufdringlich und zurückhaltend. Außerdem ist das Logo zweisprachig, wobei der englische Text größer abgedruckt ist. Unter dem Logo ist ein weiteres Logo der Fluglinie Turkish Airlines abgedruckt. Die Rundbögen spannen sich über Istanbul 2010 und somit über die ,gegenwärtige Stadt“. Das Textsegment kann dahingehend interpretiert werden, dass die (religiöse) Tradition nach wie vor über der modernen, gegenwärtigen Stadt wacht. Der Verweis auf die religiöse Tradition zieht sich damit durch: Die sakralen Bögen sind, ähnlich wie die zuvor dargestellten Turmspitzen (siehe visuelles Bild), auf höchster hierarchischer Ebene angeordnet.

Auch in diesem Schrifttyp wird durch die Kombination zweier Stilelemente „Modernität“ und „Traditionalität“" verbunden. Er ist ein Gemisch aus geschwungener, kalligrafieartiger und geradliniger, schlichter, moderner Schrift. Die mattgoldene Farbe vermittelt Eleganz. Auch hier wird als Claim der Kampagne „,inspiration“ eingeführt.

\subsection{Image-Text}

Bei der Anzeige handelt es sich um einen image-text ${ }^{6}$ im Sinne Mitchells (Mitchell 1994, S. 91). Das visuelle Bild der Stadt wird durch ein Logo, den Schriftzug ,istanbul inspirations“ und einen das Bild ,erklärenden“ Text ergänzt. Das Werbebild im Gesamten kann als sehr reduziert beschrieben werden. Auffällig ist diesbezüglich die große weiße Fläche, die das Augenmerk auf die Fotografie lenkt. Das Bild ist der „Eyecatcher“ des Werbeplakates. Besonders die zwei Moscheen links von der

\footnotetext{
${ }^{6}$ Mitchell versteht unter Image-text eine jeweils spezifische Relation zwischen Visuellem und Sprachlichem.
} 


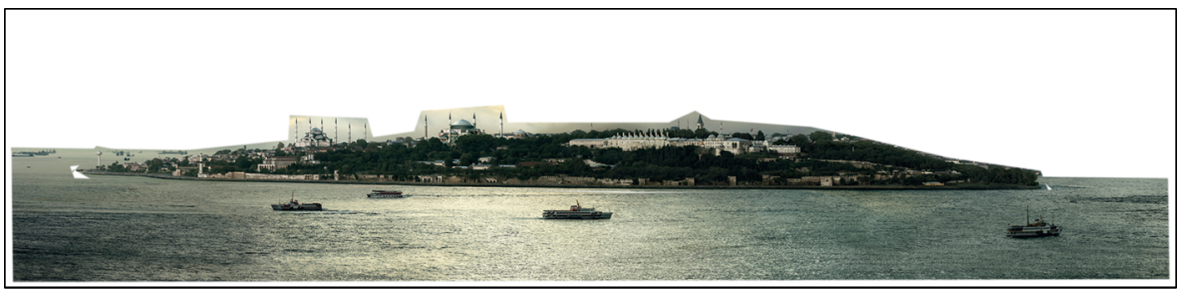

Abb. 6 Segmentzusammenhang 1 und 3 ,Wasser und Halbinsel“

Bildmitte stechen aufgrund des hell erleuchteten Hintergrunds hervor. Das Gesamtbild ist in zwei „Teile“ gegliedert: Bild und ,erklärender“ Bildtext stehen aufgrund der Position in unmittelbarer Beziehung; abgesetzt davon sind das „Logo“ (Istanbul 2010 ECOC Agency) und der „Slogan“ (istanbul inspirations). Auffallend ist nun der Kontrast zwischen dem ersten „Beziehungspaar“: dem Bild und dem das Bild „erklärenden“ Text. Der Schriftzug ist, anders als das Bild, schlicht und modern gehalten. Außerdem wird im Bild eine „malerische“ Landschaft präsentiert, die mit ihren historischen Bauwerken keineswegs an eine ,gegenwärtige“ Stadt erinnert. Neben der farblichen Gestaltung verdeckt auch der gewählte perspektivische Ausschnitt auf die Stadt gewissermaßen all jene Elemente, die dem ,modernen“ Istanbul zugeschrieben werden, wie etwa die Bosporusbrücke, der stockende Automobilverkehr, die neuen „,business improvement districts“, die Menschenmassen, die weiten Boulevards etc. Der stärker verdichtete Stadtraum befindet sich im rechten Bildhintergrund. Das Bild verweist damit ausschließlich auf das geschichtliche Istanbul. Anders verhält es sich beim Bildtext. Dieser erwähnt, dass die abgebildete Stadt ebenso durch ,contemporary art and urban culture“ inspiriert. Damit fügt der Bildtext der bildlich inszenierten „Historizität“ Istanbuls ,moderne“ Eigenschaften hinzu. Nicht nur das Pittoreske und die „Schätze“ des vergangenen Istanbuls sollen inspirieren, sondern auch das „Gegenwärtige“.

\subsection{Rekonstruktion des Entstehungs- und Gebrauchszusammenhangs}

Das hier analysierte Werbebild war das zentrale Motiv, mit dem das Kulturhauptstadtjahr 2010 international beworben wurde. Die Kampagne wurde in 14 verschiedenen Sprachen umgesetzt und in 16 Ländern beworben. Das Bild war an verschiedenen Plätzen, Einkaufsstraßen und ,gateways“ (Flughäfen, Bahnhöfen, Metrostationen etc.) in Städten wie London, Paris, Venedig, Madrid, Berlin, Frankfurt etc. auf großformatigen Bannern zu sehen. ${ }^{7}$ Ebenfalls wurde es in verschiedenen europäischen Tageszeitungen annonciert. In Deutschland waren dies beispielsweise „Die Welt“, die „Frankfurter Allgemeine“ und die „Süddeutsche Zeitung“. Außerdem war die Kampagne auf Webportalen (etwa GMX.de, TimeOut NY, Lonely Planet etc.) sowie in speziellen Magazinen wie Time, Geo, National Geographics etc. prä-

\footnotetext{
7 Diese sowie folgende Informationen zur Zielgruppe, Zielsetzung und Umsetzung bekam ich durch Interviews mit Mitarbeitern der Tourismus- und Promotion-Abteilung der Istanbul 2010 ECOC Agency, die ich im Sommer 2010 führte.
} 


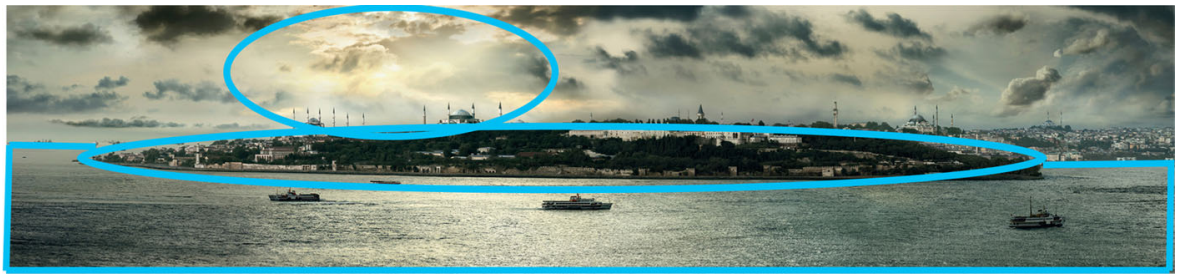

Abb. 7 „Dimensionen der Macht“

sent. Im Grunde kann man gar nicht von einem Bild sprechen. Vielmehr handelt es sich um eine aufwendige Collage, die aus 30 Fotos zusammengesetzt wurde. Die Bild-Bearbeitung dauerte drei Wochen. Dabei wurden Baukräne und andere ,störende" Elemente durch Grünflächen ersetzt (Istanbul_Magazine 2010, S. 56). Die Bilder wurden vom deutschen Fotografen Rainer Strattman aufgenommen. Darüber, was dem internationalen Publikum von Istanbul gezeigt werden sollte, bestand kein Zweifel und auch die Bedeutung des „Ikons“ wurde von den Machern sehr hoch eingeschätzt. Auf die Frage, ob die Inszenierung der geschichtsträchtigen Halbinsel der Stadt von vornherein feststand, antwortet der Leiter der Werbeagentur in einem Interview: „We had no doubts at all. This is what makes it (Istanbul) incomparable. In Venice, artists choose to iconize the Grand Canal, and now it is a must-see if you go to Venice. That is a result of that iconic photograph“ (Istanbul_Magazine 2010, S. 54). Mit der Referenz auf historische Ansichten von Venedig wird noch einmal deutlich, dass keinerlei moderne Elemente im Bild zu sehen sein sollten. Die Bosporus-Brücke wie auch die Stadt selbst werden von der Insel in der Bildmitte verdeckt. In diesem Zusammenhang spielen auch die Farben eine zentrale Rolle, wie es auch der Leiter der Werbeagentur hervorhebt: „If you pay close attention to the photos of Manhattan and London, they are both romantic and gloomy. Their most familiar photos are black and white, taken during the Second World War. Surely we have some by Ara Güler ${ }^{8}$. We wanted the colour to chime in with the eight thousand years of history“ (Istanbul_Magazine 2010, S. 54). Dementsprechend galt es bei der Gestaltung des Bildes, auf die lange Vergangenheit der Stadt Bezug zu nehmen, wobei die jüngere Vergangenheit - die Modernisierungsprozesse - sowie die Gegenwart eine weniger gewichtige Rolle spielten.

\subsection{Hegemoniale Bildpolitik}

Verweise auf ,islamische“ bzw. „osmanische“ Herrschaft finden sich an etlichen Details des Bildes: angefangen von den Minarettspitzen über den kalligrafieartigen Schrifttyp im rechten unteren Bildrand hin zum Logo der Agentur, dessen Rundbögen mit den Kuppeln von Moscheen in Verbindung gebracht werden können. Diese Überrepräsentanz von islamischen bzw. osmanischen Machtsymboliken überrascht. Denn in der Geschichte der Stadt lebten verschiedenste Religionen und Nationa-

\footnotetext{
${ }^{8}$ Güler ist einer der bekanntesten Istanbuler Fotografen, der die Modernisierung Istanbuls dokumentierte und ausschließlich in Schwarzweiß fotografiert.
} 


\section{Istanbul... the most inspiring city in the world.}

Istanbul 2010 European Capital of Culture will further inspire you

with its contemporary art and urban culture.

Be a part of this unique experience.

Abb. 8 Bildtext

litäten wie Griechen, Juden oder Armenier zusammen. Durch das Bild wird diese Pluralität alles andere als hervorgehoben. Die Machtsymbolik geht eindeutig von osmanischen Herrschaftssymbolen aus. Auch wenn bekannt ist, dass das Osmanische Reich von einem kosmopolitischen Flair umhüllt war, welches sich durch eine gewisse Toleranz gegenüber anderen Konfessionen auszeichnete, so sah das MilletSystem, die religiös definierte Rechtsordnung im Osmanischen Reich, gleichzeitig die eindeutige Vormachtstellung des Islams vor. Da die Türkei und insbesondere Istanbul eine Reihe von „Modernisierungsprozessen“ durchlaufen hat, fragt man sich, weshalb nun wieder eine ausdrückliche Bezugnahme auf die osmanische Herrschaft stattfindet. Für die hier angestellte Analyse ist das Jahr 1994 ein zentraler Wendepunkt. Mit der Machtübernahme der religiös-konservativen Partei Adalet ve Kalkınma Partisi (AKP) und speziell durch die Figur Tayyip Erdogans kam es zu einer Verschärfung des „,nostalgischen Diskurses“ (vgl. Bora 1999, Keyder 1999; Stemmler 2009). Dieser Diskurs fand in den 1950ern langsam Eingang in öffentliche Debatten und bekam ab 1994 eine politische Mehrheit. Der symbolische Stellenwert Istanbuls in den geschichtlichen Erzählungen des politischen Islams, der das Osmanische Reich und nicht die Republik als wesentlichen Bezugspunkt hat, wird von Bora wie folgt auf den Punkt gebracht:

In the popular historical narrative of both political Islam and Turkish nationalism, Istanbul is promised land. It is believed that had God wanted it, the prophet would have conquered the city; but Mohammed actually did give the good news that a great commander and his soldiers would take the city for Islam. Istanbul thus attains holiness, both because the prophet predicted its conquest and because it served as the capital of the Islamic Ottoman Empire. Istanbul is the Islamic city, the jewel of the Islamic universe. (Bora 1999, S. 48; Hervorh. i. O.)

Vor allem mit der Gründung der Republik und der darauffolgenden Modernisierung verlor Istanbul an politischer, kultureller und wirtschaftlicher Bedeutung: ,(...) by this time the city had lost most of its cosmopolitan character; it had become simply the primate city of a rather poor and isolated nation-state" (Keyder 1999, S. 11). Das neue Zentrum war Ankara. Istanbul wurde von der dortigen Elite mit Argwohn betrachtet: ,as a den of corruption and intrigue with ambivalent allegiance to the nationalist project" (ebd.). Im islamischen Diskurs verlor die Stadt jedoch über die ,reale“ Bedeutung hinaus ,,it's soul and beauty“ (Bora 1999, S. 48). Bereits seit den 1950ern zweifelten sowohl islamische als auch konservativ-nationalistische Positionen an der Modernisierung und es wurde die Meinung vertreten, dass Istanbul erneut ,erobert“ werden müsse (vgl. ebd.), damit die Stadt wieder zu dem 
Abb. 9 „Logo European Capital of Culture Agency“

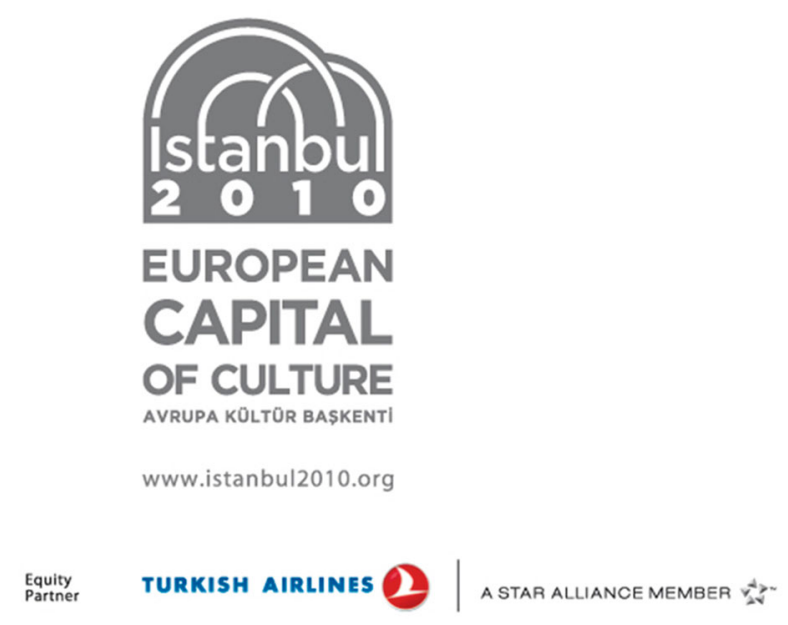

glänzenden Juwel würde, den sie einmal verkörperte. Schon vor seiner Machtübernahme sprach Tayyip Erdogan in der Tageszeitung Hurrieyet (26. 12. 1993) über die zweite „Eroberung“ der Stadt (,second taking of Istanbul“) (vgl. ebd.). Die durch die Modernisierungsprozesse angestoßenen Veränderungen der Stadt wurden seitens konservativer Positionen als erneute Vereinnahmung des Westens gedeutet. Dem sollte seit der Machtübernahme Erdogans ein symbolisches Ende bereitet werden. Nicht nur der Stadtbevölkerung, sondern auch den Touristen wurde dieser, milde formuliert, „Wandel“ vor Augen geführt. Um die Identität Istanbuls als ,islamische Stadt“ zu stärken, wurde von Erdogan der Bau einer Moschee und eines islamischen Kulturzentrums im „modernen“ Teil der Stadt (Taksim) entschieden und wie folgt argumentiert:

This is the point of attraction of Istanbul's tourism. The person who comes here should be able to tell that he has arrived in an Islamic city ... As we succeed in uncovering the historical and cultural texture of our city, its Muslim character will become apparent to the visitor (Erdogan 1994, zit. nach Bora 1999, S. 49).

Die politischen und islamischen Machtsymboliken sind also nicht zufällig auch in dem hier vorgeführten Werbebild zu finden. Die AKP machte sich die visuelle Kommunikation Istanbuls während des Kulturhauptstadtjahres zu Nutze (Göktürk 2012). Die Initiative zur Bewerbung zum Kulturhauptstadtjahr ging zwar von einer Reihe unabhängiger Organisationen aus dem Kulturbereich aus. Ebenfalls im Bewerbungskonzept wurde die partizipative und demokratische Umsetzung des Events stark gemacht. Als jedoch die offizielle Organisation „Istanbul 2010 ECOC Agency“ 2007 gegründet wurde, kam es zu einer Umstrukturierung der Initiative, womit die Politik die Entscheidungskompetenz an sich riss:

The law that enabled the establishment of the Agency called for a strictly bureaucratic and hierarchical structure, directly connected to the Office of the Prime Minister. (...) Decision-making slowly shifted from the actors represen- 


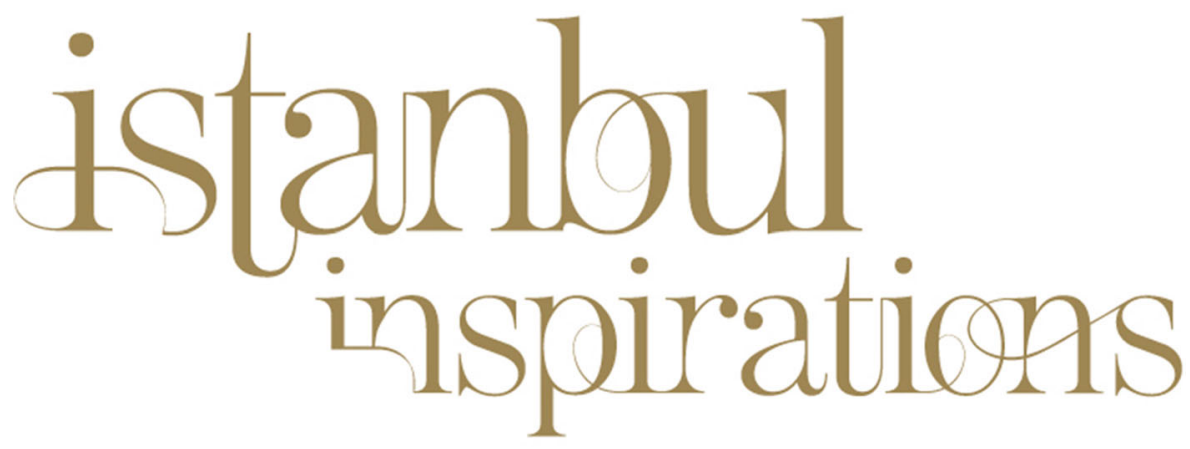

Abb. 10 Slogan der internationalen Werbekampagne

ting civic bodies to the governmental authorities; the decisions about which projects to fund were increasingly made by the governmental representatives (Öner 2010, S. 270).

Damit änderte sich auch das inhaltliche Programm des Kulturhauptstadtjahres, bei dem nun weniger die Präsentation der jungen Kulturszene Istanbuls im Vordergrund stand als die Restaurierung und Repräsentation historischer und vor allem osmanischer Bauwerke (Hein 2010). Die Umstrukturierung der Initiative und die mit ihr einhergehenden thematischen Verschiebungen der Ausrichtung des Kulturhauptstadtjahres machen sich in dem hier vorgestellten Werbebild bemerkbar.

\subsection{Nostalgische Ansicht}

Wie schon bei der Ersteindrucksanalyse unterschiedlicher Betrachterinnen aufgefallen war, ist auch eine zweite, etwas differente Wahrnehmung des Bildes möglich. Neben den visualisierten Machtsymboliken ruft das Bild märchenhafte und stimmungsvolle Assoziationen hervor. Man hat das Gefühl, in eine vergangene Zeit zurückversetzt zu sein und in eine ,andere“ Welt einzutauchen. Es wird eine Exotisierung vorgenommen. Dabei gilt es zu bestimmen, ob und wie sich die Istanbuler Bevölkerung selbst mit dem westlichen Blick der ,otherisation“ (Eldem 2010) wahrnimmt. Nun muss zu allererst festgehalten werden, dass die Osmanen vielfach Zielscheibe orientalistischer Zuschreibungen waren, ,a Western intellectual construct of essentialist otherisation" (Eldem 2010, S. 27). Abgesehen davon, ob positive oder negative Attribute zugewiesen wurden, ging es um die Konstruktion eines absoluten Anderen. Orientalistische Zuschreibungen kennzeichneten eine Hassliebe und schwankten zwischen Bewunderung und Abscheu. „Ottomans were barbarians who had to civilise (Renan), but their aping of the West meant the destruction of their exotic and somewhat noble self (Loti). Damned if you do, damned if you don't" (ebd., S. 27). Vor allem während der Modernisierungsprozesse seit 1923 grenzte sich die türkische Elite von den vom Westen auf die Osmanen projizierten Attributen ab, in dem man diese auf spezifische ethnische und religiöse Gruppen der Türkei übertrug und westliche Standards für sich selbst beanspruchte. Mit der Entstehung 


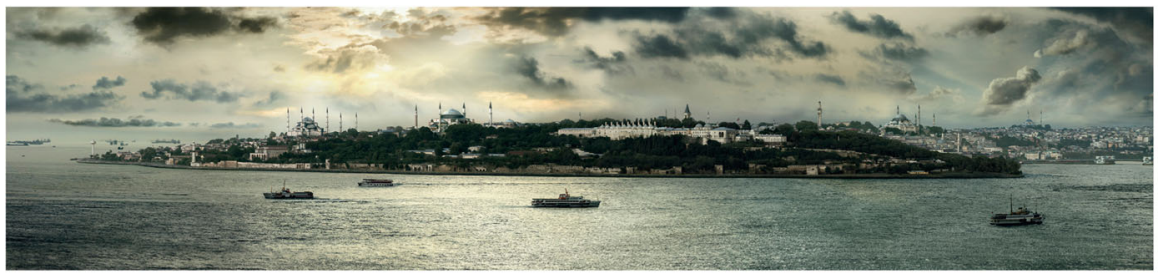

Istanbul... the most inspiring city in the world. Istanbul 2010 European Capital of Culture will further inspire you
with its contemporary art and urban culture. Be a part of this unique experience
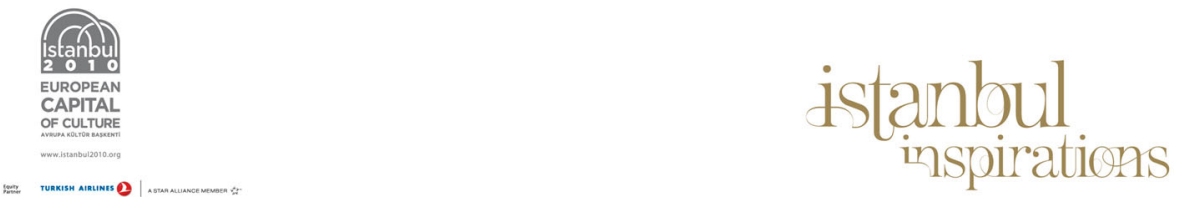

Abb. 11 Gesamter, ,image-text“

des zuvor beschriebenen „nostalgischen Diskurses“, der unter anderem auf ein neu aufkommendes Selbstbewusstsein religiös-konservativer Kreise zurückzuführen ist, wurden Symboliken der osmanischen Kultur wieder reaktiviert. Zunächst wurde im touristischen Bereich erneut mit vergangenen Zuschreibungen geworben, da internationale Besucher gewisse Erwartungen an eine „orientalische Stadt“ hatten: „The main external challenge took the form of tourism and its expectation of an Oriental appeal rather than a show of peripheral modernity“ (Eldem 2010, S. 29). Schließlich fing man jedoch an, dieselbe „Exotik“ zu konsumieren; eine Form postmoderner „self-exoticisation“ (ebd., S. 31). Die Übernahme des westlichen Blicks erfolgte also in drei Schritten: Abgrenzung, Vermarktung, Internalisierung. Diese Verstrickung der Zuschreibungen von außen und der eigenen Selbstwahrnehmung der Istanbuler Elite bringt Orhan Pamuk in seinem Werk „Istanbul - Erinnerungen an eine Stadt“ zum Ausdruck. Darin finden sich zahlreiche intertextuelle Verweise auf Schilderungen der Stadt aus der Sicht europäischer Schriftsteller und Reisender (etwa Gérard de Nerval, Gustave Flaubert, Théophile Gautier und Pierre Loti). Diese vermischen sich mit den Darstellungen Istanbuler Literaten (Yahya Kemal, Ahmet Rasim, Resat Ekrem Kocu), dem innerstädtischen Diskurs und eigenen autobiographischen Erfahrungen. Pamuk kommt zum Schluss, dass die Außenperspektive europäischer Reisender, insbesondere von Schriftstellern und Malern, die Selbstwahrnehmung der Istanbuler stark beeinflusste und sie gar mit deren Augen auf ihre Stadt blicken:

Tatsache ist, daß wir alle einen teils verborgenen, teils offen lesbaren Text im Kopf haben, der allem was wir tun und lassen irgendwie einen Sinn verleiht. Und in diesem Text nimmt das, was westliche Beobachter über uns sagen, breiten Raum ein. (...) Und wenn ich das Gefühl habe, daß gerade keine westlichen Augen auf mir ruhen, werde ich eben selbst zu meinem eigenen Westler (Pamuk 2008, S. 327 f.). 


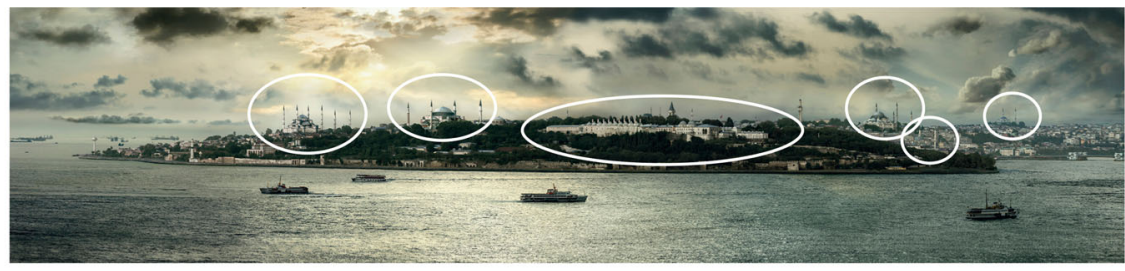

Istanbul... the most inspiring city in the world, Istanbul 2010 European Capitial of Culture will further inspire you
with is Be a part of this unique experience
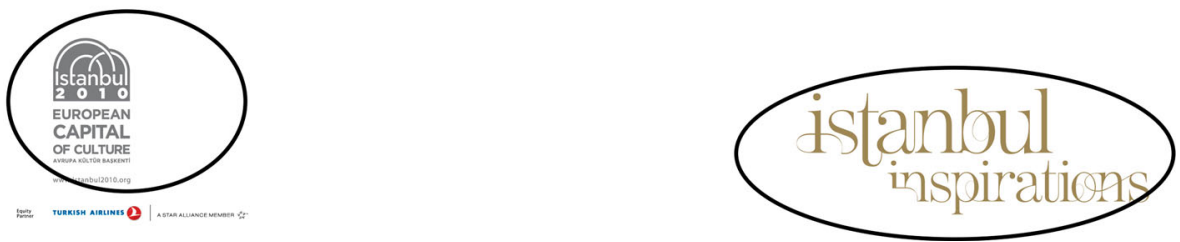

Abb. 12 Islamisch/osmanische Symbole im Bild

Nun blicken die von Pamuk rezipierten europäischen Autoren mit einem melancholischen Blick auf die Stadt. Was sie an ihr lieben, ist die von Modernisierungsprozessen verschont gebliebene Stadt. Die Inspiration, die sie in der Stadt finden, ist jene, die sie zeitlich zurückversetzt und an den Geschichten, die über diese ,,andere Welt" erzählt werden, anschließt. Vor allem Pierre Loti klagt über die Gefahr des Verlustes des pittoresken Orients, einer ,alten“ Türkei, die der modernen weichen musste (Stemmler 2009, S. 297). Die nostalgische Melancholie entsteht durch diese im Verschwinden begriffene Schönheit.

Der Reiz, den der „Orient“ ausstrahlt, ist immer der vergangene. Dem Anderen wird im Betrachten eine Zeitstelle zugewiesen; noch ist es da, und wenn man es nicht mit dem eigenen Blick einfängt, dann droht es zu verschwinden. Das Verschwinden des Anderen ist aber geradezu die Bedingung für den visuellen und körperlichen Genuss (ebd., S. 296).

Diese Nostalgie wird durch das analysierte Werbebild konstruiert und schafft dadurch seinen Reiz sowohl für das internationale Publikum als auch für die Istanbuler Bevölkerung. Durch das gewählte Medium, der einer Malerei ähnelnden Fotografie, wird dieser Akt des Verschwindens nochmals verstärkt (vgl. Barthes 1989, S. 106).

\subsection{Zusammenfassung der Analyse des Werbebildes}

Das Bild deutet darauf hin, dass religiöse Macht, wie in Zeiten des Osmanischen Reiches, eng mit der politischen Führung verbunden ist. Das ,weltliche“ Herrschaftszentrum in Gestalt des Topkapi-Palastes wird von beiden Seiten von Moscheen umstellt. Durch die Analyse wurde eine Visualisierung hegemonialer Machtansprüche erkannt. Mit Verweis auf Aussagen der gegenwärtigen politischen Führung und mit Einbezug kritischer Evaluationen des Kulturhauptstadtjahres, die eine starke Ein- 
flussnahme der politischen Führung bei der Gestaltung der Kampagnen konstatierten, wurde bemerkt, dass im Bild mitunter eine „Wiedereroberung“ dieser Vergangenheit Istanbuls zur Schau gestellt werden soll. Wie weit man mit solchen Einschätzungen auch gehen mag, klar am Bild erkennbar ist ein Bruch mit jenen Modernisierungsbestrebungen seit der Gründung der Republik, die sich vom Osmanischen Reich abgrenzten, und eine eindeutige Wiederbelebung der „Belle Epoque“ (Fuhrmann 2010, S. 19), als Istanbul noch Empire war. Diese Rückbesinnung ist gleichzeitig an einem stärker werdenden ,nostalgischen Diskurs“ in Istanbul erkennbar (vgl. Bora 1999, S. 49). Jene Vergangenheit passt insofern zu den ,postmodernen Werten“ einer ,globalen“ Stadt, als diese Epoche Inbegriff kosmopolitischer, synkretistischer, pluralistischer und moderner Werte ist (Tischler 2010, S. 112). Ebenfalls ist dieses Image für eine Städtereisedestination attraktiv, da die Zeit der Osmanen mit exotischen erotischen Vorstellungen assoziiert wird. Das Bild suggeriert eine ,fremde Welt“, die es zu entdecken gilt und die den ,Westler“" inspirieren wird. Das analysierte Werbebild verflechtet die hegemoniale islamische Machtsymbolik mit dem nostalgischen Diskurs. Dadurch könnte die Macht hinterfragt werden, denn es ist ja klar, dass der Topkapi-Palast heute nur ein Museum ist, eine touristische Attraktion und keineswegs ein gegenwärtiges Zentrum politischer Macht. Der starke Vergangenheitsbezug lässt die Bildbetrachterin zweifeln, ob ein derartiges Reich noch existieren kann, und wenn es dieses noch gibt, wie das Foto suggeriert, dann ist es im Verschwinden begriffen. Es kann sich nicht länger halten, da die Welt heute nach anderen Regeln funktioniert. Das Werbeimage funktioniert jedoch wie ein Vexierbild, das entweder die hegemoniale Machtdarstellung oder die nostalgische Ansicht Istanbuls hervortreten lässt. Es stärkt zugleich die lokale Verbundenheit und erweckt globale Neugier. Wären etwa nur hegemoniale Machtsymboliken erkennbar, würde dies Touristen wohl eher abschrecken. Durch die Verschränkung beider „Logiken“ wird das bildspezifische Potenzial genutzt, Gegensätzliches kongruent erscheinen zu lassen (Bohnsack 2009) und eine enorme Geschehensdichte aufzubauen (Imdahl 1994). Eine bestimmte Epoche der Vergangenheit wird zur Darstellung des neu erstarkten Selbstbewusstseins Istanbuls in Teilen seiner politischen und wirtschaftlichen Eliten und als zukunftsträchtiges Image einer postmodernen „Global City“ stilisiert. Die breite Masse internationaler Touristinnen wird in ihrem „westlichen Blick“ von Istanbul bestätigt, welcher potenzielle negative Attribute ins Positive übersetzt wie etwa: „rückständig, aber authentisch“. Von diesem Blick eingenommen, bleibt die stark ausgeformte Machtsymbolik unerkannt.

\section{Fazit: Bildpolitik mit Stadtimages}

Stadtimages können als „Kompaktimpressionen“ (Marent et al. 2014) verstanden werden. Sie bringen eine überdeterminierte und polysemische Wirklichkeit der Stadt kondensiert zum Ausdruck. Die Analyse hat gezeigt, dass Werbebilder alles andere als unterkomplex sind und als Wirkungsgefüge aus gesellschaftlicher Sichtbarkeit, Genreästhetik, Produktionskontexten und lokalen Diskursen zu beschreiben sind. Stadtbilder, wie das hier analysierte Werbebild Istanbuls, zeigen die Stadt nicht nur wie sie ist, sondern erzeugen visuelle Vorstellungswelten, die wiederum handlungs- 
strukturierend wirken. In dieser Hinsicht wurde beschrieben, wie Herrschaftssymboliken im Bild zum Ausdruck kommen und weshalb der Wandel der Stadt durch die Wiederbelebung der Vergangenheit zum Ausdruck gebracht wird. Das Bedeutungspotenzial des Bildes wurde an der Verschachtelung von hegemonialem Machtanspruch und dem Rückbezug auf eine bestimmte Epoche der Vergangenheit festgemacht. Ebenfalls wurde darauf geachtet, welche Bedeutungsverwerfungen bzw. -verschiebungen durch die bildimmanenten Verdichtungsleistungen vorgenommen werden. Erkennbar sind diesbezüglich die Abwendung von der jüngeren Vergangenheit und die Ausblendung moderner Aspekte der Stadt. Die Analyse hat gezeigt, dass der Symbolkosmos Istanbul nicht hermetisch geschlossen ist. Das hier analysierte Bild greift auf ,andere“ bzw. „fremde“ Bilder zurück. Analogien zu europäischen Stadtdarstellungen wie die flächige Panoramaperspektive, die Überhöhung der Sakralbauten und die Darstellung der Stadt in Form einer Ellipse sind deutlich im Bild erkennbar. Auch der Leiter der Werbeagentur sprach in einem Interview explizit an, von welchen Bildern man sich abgrenzen wollte, nämlich den klassischen Ansichten von Manhattan und London, und welche Stilisierungen man aufgegriffen hat mit Blick auf historische Ansichten des Canal Grande in Venedig. Europa bzw. die ,europäische Stadt“ (Häußermann 2001) und die mit diesen Begriffen verbundenen Urbanitätsvorstellungen sowie die Bilder und Narrative, die von reisenden Malern und Schriftstellern erstellt wurden, durchzogen als Referenz das hier analysierte Bild. Dennoch, und das sollte durch die hier vorgestellte Analyse deutlich geworden sein, werden diese ,äußeren“ Einflüsse lokalspezifisch verarbeitet und das Werbebild kann als städtisches Selbstbild begriffen werden: ${ }^{9}$ Istanbul steht in einem Spannungsverhältnis zwischen Fremd- und Selbstwahrnehmung, enormem Wandel bei gleichzeitiger Reaktivierung der Vergangenheit und einer Machtdarstellung, die gerade durch ihre überdeutliche Darstellung brüchig erscheint.

Open Access This article is distributed under the terms of the Creative Commons Attribution 4.0 International License (http://creativecommons.org/licenses/by/4.0/), which permits unrestricted use, distribution, and reproduction in any medium, provided you give appropriate credit to the original author(s) and the source, provide a link to the Creative Commons license, and indicate if changes were made.

\section{Literatur}

Barthes, Roland. 1989. Die helle Kammer. Bemerkungen zur Photographie. Frankfurt a. M.: Suhrkamp. Berger, John. 1974. Sehen. Das Bild der Welt in der Bilderwelt. Hamburg: Rowohlt.

Berking, Helmuth und Martina Löw. 2008. Die Eigenlogik der Städte. Neue Wege für die Stadtforschung. Frankfurt a. M.: Campus.

Berking, Helmuth und Jochen Schwenk. 2011. Hafenstädte. Bremerhaven und Rostock im Wandel. Frankfurt a. M.: Campus.

Bohnsack, Ralf. 2009. Qualitative Bild- und Videointerpretation. Die dokumentarische Methode. Opladen, Farmington Hills: Babara Budrich.

Bora, Tanil. 1999. Istanbul of the Conqueror. The Alternative Global City. Dreams of Political Islam. In Istanbul between the Global and the Local, Hrsg. Caglar Keyder, 47-58. Boston: Rowmann \& Littlefield.

Breckner, Roswitha. 2010. Sozialtheorie des Bildes: Zur interpretativen Analyse von Bildern und Fotografien. Bielefeld: Transcript.

\footnotetext{
9 Dieses Spannungsverhältnis wird in der Arbeit „Istanbul als Bild. Eine Analyse urbaner Vorstellungswelten" genauer beschrieben (Marent 2016).
} 
De Seta, Cesare. 1999. Eine deutsche Städteikonographie in europäischer Perspektive. In Das Bild der Stadt in der Neuzeit. 1400-1800, Hrsg. Wolfgang Behringer, Bernd Roeck, 1-12. München: C. H. Beck.

Eldem, Edhem. 2010. Ottoman and Turkish Orientalism. Architectural Design 80(1):26-31.

Frank, Sybille, Michael Haus, Petra Gehring, et al. 2014. Städte unterscheiden lernen zur Analyse interurbaner Kontraste: Birmingham, Dortmund, Frankfurt, Glasgow. Frankfurt am Main: Campus-Verl.

Fuhrmann, Malte. 2010. Vom stadtpolitischen Umgang mit dem Erbe der Europäisierung in Istanbul, Izmir und Thessaloniki. Frankfurt a. M.: Peter Lang.

Goffman, Erving. 1980. Rahmen-Analyse: Ein Versuch über die Organisation von Alltagserfahrungen. Frankfurt a. M.: Suhrkamp.

Goffman, Erving. 1981. Geschlecht und Werbung. Frankfurt a. M.: Suhrkamp.

Goffman, Erving. 1986. Interaktionsrituale. Über Verhalten in direkter Kommunikation. Frankfurt am Main: Suhrkamp.

Göktürk, Deniz. 2012. Orientierte Vielfalt. Hauptstadt Europa - von der Ruhr bis an den Bosporus? In Verortungen der Interkulturalität. Die Europäischen Kulturhauptstädte Luxemburg und die Großregion (2007), das Ruhrgebiet (2010) und Istanbul (2010), Hrsg. Thomas Ernst, Dieter Heimböckel, 221-246. Bielefeld: Transcript.

Günther, Lutz Philipp. 2009. Die bildhafte Repräsentation deutscher Städte. Von den Chroniken der Frühen Neuzeit zu den Websites der Gegenwart. Köln, Weimar, Wien: Böhlau Verlag.

Häußermann, Hartmut. 2001. Die europäische Stadt. Leviathan. Berliner Zeitschrift für Sozialwissenschaft 29(2):237-255.

Hein, Carola. 2010. The European Capital of Culture Programme and Istanbul 2010. In Orienting Istanbul. Cultural Capital of Europe?, Hrsg. Deniz Göktürk, Levent Soysal, Ipek Türeli, 253-266. London, New York: Routledge.

Imdahl, Max. 1994. Ikonik. Bilder und ihre Anschauung. In Was ist ein Bild?, Hrsg. Gottfried Boehm, 300-324. München: Wilhelm Fink Verlag.

Istanbul_Magazine. 2010. This photo will be an icon of Istanbul forever. Istanbul 2010 1:54-59.

Keyder, Caglar. 1999. The Setting. In Istanbul between the Global and the Local, Hrsg. Caglar Keyder, 3-30. Boston: Rowmann \& Littlefield.

Lindner, Rolf. 2008. Textur, imaginaire und Habitus. Schlüsselbegriffe der kulturanalytischen Stadtforschung. In Die Eigenlogik der Städte. Neue Wege für die Stadtforschung, Hrsg. Helmuth Berking, Martina Löw, 83-94. Frankfurt a. M.: Campus.

Löw, Martina. 2008. Soziologie der Städte. Frankfurt a. M.: Suhrkamp.

Marent, Johannes. 2016. Istanbul als Bild. Eine Analyse urbaner Vorstellungswelten. Bielefeld: Transcript.

Marent, Johannes und Christoph Rosenbusch. 2014. Rhythmik in Bildern: Kompaktimpressionen. In Städte unterscheiden lernen. Zur Analyse interurbaner Kontraste: Birmingham, Dortmund, Frankfurt, Glasgow, Hrsg. Sybille Frank, Petra Gehring, Julika Griem, et al., 69-96. Frankfurt a. M.: Campus.

Michalski, Sergiusz. 1999. Vom Himmlischen Jerusalem bis zu den Veduten des 18. Jahrhunderts - Symbolik und Darstellungsparadigmen der Stadtprofilansichten. In Das Bild der Stadt in der Neuzeit. 1400-1800, Hrsg. Wolfgang Behringer, Bernd Roeck, 46-55. München: C. H. Beck.

Mitchell, Thomas W.J. 1994. Picture theory. Essays on verbal and visual representation. Chicago: University of Chicago Press.

Müller-Doohm, Stefan. 1997. Bildinterpretation als struktural-hermeneutische Symbolanalyse. In Sozialwissenschaftliche Hermeneutik. Eine Einfuihrung, Hrsg. Ronald Hitzler, Anne Honer, 81-108. Opladen: Leske + Budrich.

Öner, Oguz. 2010. Istanbul 2010 European Capital of Culture: Towards a Participatory Culture? In Orienting Istanbul. Cultural Capital of Europe?, Hrsg. Deniz Göktürk, Levent Soysal, Ipek Türeli, 267-278. London, New York: Routledge.

Pamuk, Orhan. 2008. Istanbul: Erinnerungen an eine Stadt. Frankfurt a. M.: Fischer.

Raab, Jürgen. 2012. Visuelle Wissenssoziologie der Fotografie. Sozialwissenschaftliche Analysearbeit zwischen Einzelbild, Bildkontexten und Sozialmilieu. ÖZS 37(2):121-142.

Richter, Ralph. 2014. Differenzierung inszenieren: Der Fall Stadtmarketing. In Städte unterscheiden lernen. Zur Analyse interurbaner Kontraste, Hrsg. Sybille Frank, Petra Gehring, Julika Griem, et al., 246-281. Frankfurt, New York: Campus.

Stemmler, Susanne. 2009. Stadtplan als Autobiografie. Orhan Pamuks melancholischer Blick auf Istanbul. In Metropolen im Maßstab. Der Stadtplan als Matrix des Erzählens in Literatur, Film und Kunst, Hrsg. Achim Hötler, Volker Pantenburg, Susanne Stemmler, 289-299. Bielefeld: Transcript.

Strauss, Anselm L. 1961. Images of the American City. New York: The Free Press of Glencoe. 
Tischler, Ulrike. 2010. Der Sonderfall Beyoglu: Ikonographie eines Begegnungsraumes zwischen Ost und West. In Bilderwelten - Weltbilder. Die Gegenwart der Vergangenheit in postosmanischen Metropolen Südosteuropas: Thessaloniki, Istanbul, Izmir, Hrsg. Ulrike Tischler, Ioannis Zelepos, 101-160. Frankfurt a. M.: Peter Lang.

Wietschorke, Jens. 2013. Anthropologie der Stadt: Konzepte und Perspektiven. In Stadt. Ein interdisziplinäres Handbuch, Hrsg. Harald A. Mieg, Christoph Heyl, 202-221. Stuttgart, Weimar: J. B. Metzler.

Willems, Herbert und York Kautt. 2003. Theatralität der Werbung. Theorie und Analyse massenmedialer Wirklichkeit: Zur kulturellen Konstruktion von Identitäten. Berlin, New York: Walter de Gruyter.

Zurstiege, Guido. 2007. Werbeforschung. Konstanz: UVK.

Johannes Marent ist Lektor am Institut für Soziologie der Universität Wien. Er promovierte 2015 an der Universität Wien mit der Arbeit „Istanbul als Bild. Eine Analyse urbaner Vorstellungswelten“. Von 2011 bis 2014 war er Mitarbeiter im DFG-Projekt „Die Inszenierung des Ganzen: Stadtmarketing und die Eigenlogik der Städte“ am Institut für Soziologie der TU Darmstadt. Seine Forschungsschwerpunkte sind Visuelle Soziologie, Stadtforschung, Kultursoziologie, Soziale Medien und Migration. Er ist Mitglied im Forschungsnetzwerk ,Visual Studies in den Sozialwissenschaften“ der Universität Wien (visualstudies.univie.ac.at) 